\title{
Magnetics Influence for Calculating the Structural and Thermodynamic Properties of $\sigma$-phase in the Fe-V System for Three-sub-lattice Model
}

\section{A. L. Udovsky and M. V. Kupavtsev}

Baikov Institute of Metallurgy and Materials Science, Russian Academy of Sciences, Russia, 119991, Moscow, Leninsky avenue, 49

\section{Abstract}

The three-sub-lattice approximation model calculations of the energies and magnetic parameters (Curie temperature and mean magnetic moment) were used. The distributions of atomic species between different model sub-lattices and the thermodynamic properties depending on the $\sigma \mathrm{Fe}-\mathrm{V}$ alloy compositions at $\mathrm{T}=\mathrm{oK}$

Corresponding Author: A. L. Udovsky

Received: 21 December 2017

Accepted: 15 April 2018

Published: 6 May 2018

Publishing services provided by Knowledge $\mathrm{E}$

(c) A. L. Udovsky and M. V. Kupavtsev. This article is distributed under the terms of the Creative Commons

Attribution License, which permits unrestricted use and redistribution provided that the original author and source are credited.

Selection and Peer-review under the responsibility of the MIE-2017 Conference Committee.

\section{G OPEN ACCESS} were evaluated by solving the set of equations at $\mathrm{T}=\mathrm{oK}$ obtained by minimizing the functional $\Delta G$ with respect to the independent configuration degrees of freedom. The comparison of the calculated data on the structural properties of $\sigma$-phases in $\mathrm{Fe}-(\mathrm{Cr}, \mathrm{V})$ systems depending on composition and temperature was conducted. The consistency of the input data and the calculation results for the thermodynamic properties is obtained, namely, the dependence of the enthalpy of mixing of the $\sigma$-phase of the Fe-V system on the composition relatively of the BCC phases of pure $\mathrm{Fe}$ and $\mathrm{V}$. The input data was used to find the initial values of the model parameters. The consistency of these data proves the correctness of the found energy parameters of the model.

Keywords: thermodynamics; three-sub-lattice model; Sigma - phase; alloys for nuclear energy; Fe-V, Fe-Cr systems.

\section{Introduction}

Previously, the three-sub-lattice model ( $3 S M$ ) was used by the authors for a consistent description of the structural properties (SP) and thermodynamic properties of the Fe$\mathrm{Cr} \sigma$-phase $[1,2]$. The difference between the $\mathrm{Fe}-\mathrm{Cr}$ and $\mathrm{Fe}-\mathrm{V}$ systems consists in the fact that the $\sigma$-phase in the Fe-V system is stable both in the ferromagnetic state and paramagnetic state. The Curie temperature (Tc) for the $\sigma \mathrm{Fe}-\mathrm{V}$ alloys is an order of magnitude higher than $\mathrm{Tc}$ for the $\sigma \mathrm{Fe}-\mathrm{Cr}$ alloys, according to experimental data [3]. Therefore, the $\sigma$-phase in this system was considered in the paramagnetic state. In the 
framework of the physic-empirical model, the configuration contribution and magnetic contribution to the Gibbs mixing energy $(\Delta \mathrm{G})$ for the sigma phase in the Fe-V system was taken into account.

\section{Formulation of the model}

The crystal lattice of sigma-phase contains 5 sub-lattices in which 30 atoms possessing of different coordination numbers of the nearest neighbors (12, 14 and 15) are placed. In [1] the real structure of sigma-phase modeled with 3 sub-lattices to describe the distribution of atoms inside the structure of sigma-phase phase depending on the alloy composition and temperature. According to $3 \mathrm{SM}$ the real structure of $\sigma$-phase $\mathrm{A}_{2}{ }^{12} \mathrm{~B}_{4}{ }^{15} \mathrm{C}_{8}{ }^{14} \mathrm{D}_{8}{ }^{12} \mathrm{E}_{8}{ }^{14}$ (including 5 sub-lattices) was replaced by 3 sub-lattices. All of the model sub-lattices are being filled by atoms of two species with coordination numbers $(12,14$ and 15$)$. The method of calculation of structural and thermodynamic properties of $\sigma$-phases within 3SM was described in [1-2], and applied to the $\mathrm{Fe}-\mathrm{Cr}$ system, where the energy parameters were calculated by "fixing" to the results of quantum mechanical calculations obtained at oK. The functional of free energy mixing for sigmaphase relatively the $\mathrm{BCC}$-phases of the two species within the $3 \mathrm{SM}$ is presented in the following form:

$$
\begin{aligned}
& { }^{f B C C} \Delta^{p B C C} G^{\sigma}\left(x_{V}, y_{2}^{12}, y_{2}^{15}, T\right)=x \cdot \delta E_{1}+y_{2}^{12} \cdot \delta E_{2}+y_{2}^{14} \cdot \delta E_{3} \\
& +x^{2} \cdot \delta E_{4}+\left(y_{2}^{12}\right)^{2} \cdot \delta E_{5}+\cdot\left(y_{2}^{14}\right)^{2} \cdot \delta E_{6}+x y_{2}^{12} \cdot \delta E_{7}+x y_{2}^{14} \cdot \delta E_{8}+y_{2}^{12} y_{2}^{14} \cdot \delta E_{9}+(1-x) \\
& \cdot\left(\Delta H_{F e}^{f \sigma \rightarrow f B C C}-T \Delta S_{F e}^{f \sigma \rightarrow f B C C}\right)+ \\
& +x \cdot\left(\Delta H_{V}^{p \sigma \rightarrow f B C C}-T \cdot \Delta S_{V}^{p \sigma \rightarrow f B C C}\right)+R T \cdot\left\{\ln \left(B_{0}(x)+1\right)\right\} \cdot g(\tau(x))+ \\
& +R \cdot T \cdot\left[\begin{array}{l}
\alpha^{12}\left(y_{1}^{12} \cdot \ln \left(y_{1}^{12}\right)+y_{2}^{12} \cdot \ln \left(y_{2}^{12}\right)\right)+ \\
\alpha^{14}\left(y_{1}^{14} \cdot \ln \left(y_{1}^{14}\right)+y_{2}^{15} \cdot \ln \left(y_{2}^{14}\right)\right)+ \\
\alpha^{15}\left(y_{1}^{15} \cdot \ln \left(y_{1}^{15}\right)+y_{2}^{15} \cdot \ln \left(y_{2}^{15}\right)\right)
\end{array}\right]
\end{aligned}
$$

where

$$
\begin{gathered}
g(\tau(x))=1-\frac{\left[\frac{79 \cdot \tau^{-1}}{140 \cdot p}+\frac{474}{497} \cdot\left(\frac{1}{p}-1\right) \cdot\left(\frac{\tau^{3}}{6}+\frac{\tau^{9}}{135}+\frac{\tau^{15}}{600}\right)\right]}{D}, \quad \tau(x)=\frac{T}{T_{c}(x)} \\
a^{12} y_{2}^{12}+a^{14} y_{2}^{14}+a^{15} y_{2}^{15}=30 \cdot x ; \quad \alpha^{12}=10 / 30 ; \quad \alpha^{14}=16 / 30 ; \quad \alpha^{15}=4 / 30,
\end{gathered}
$$


where $x$ - composition of the second species $(\mathrm{V})$ in the Fe-V system. $y_{1}^{i}, y_{2}^{i}-(\mathrm{i}=12$; $14 ; 15)$ mole fractions of the first and second species in the 3SM of the $\sigma$-phase. The $y_{2}^{12}=y_{V}^{12}, y_{2}^{14}=y_{V}^{14}$ selected as independent variables, and $x=x_{V}$ the vanadium concentration parameter.

The values of the internal parameters of the model (with a constant composition and temperature) are found from the solution of the set of equations (3) and the local stability conditions (3b)

$$
\begin{gathered}
\left\{\begin{array}{l}
\frac{d^{\sigma} \Delta^{\sigma} G^{\sigma}(x, T)}{d y_{2}^{12}}=0 \\
\frac{d^{\sigma} \Delta^{\sigma} G^{\sigma}(x, T)}{d y_{2}^{15}}=0
\end{array}\right. \\
\operatorname{det}\left\|\begin{array}{ll}
\frac{d^{2} \Delta^{\sigma} G^{\sigma}(x, T)}{d y_{2}^{(12)^{2}}} \frac{d^{2} \Delta^{\sigma} G^{\sigma}(x, T)}{d y_{2}^{(15)} d y_{2}^{(12)}} \\
\frac{d^{2} \Delta^{\sigma} G^{\sigma}(x, T)}{d y_{2}^{(12)} d y_{2}^{(15)}}
\end{array} \frac{d^{2} \Delta^{\sigma} G^{\sigma}(x, T)}{d y_{2}^{(15)^{2}}}\right\|>0
\end{gathered}
$$

Conditions of the local and global sustainability can be rewritten as:

$$
G e s \Delta H^{\sigma}\left(x_{V}, y_{2}^{12}, y_{2}^{14}, 0\right)=\left|\begin{array}{ccc}
2 \cdot \delta E_{5} & \delta E_{9} & \delta E_{7} \\
\delta E_{9} & 2 \cdot \delta E_{6} & \delta E_{8} \\
\delta E_{7} & \delta E_{8} & 2 \cdot \delta E_{4}
\end{array}\right|>0 ; \quad 4 \cdot \delta E_{5} \cdot \delta E_{5}-\left(\delta E_{9}\right)^{2}>0
$$

\section{The calculations of energetic parameters}

The problem of calculating the energetic parameters of $3 \mathrm{SM}$ was due to the fact that no evidence of ab-initio calculations of enthalpy of mixing in the system Fe-V. To solve this problem, the authors of this paper propose a novel approach. Use the features of the phase diagram of Fe-V (see Fig.1) [1], namely a point of equal concentrations (PEC) between $\sigma$-and bcc- phases at T $=1750 \mathrm{~K}$. Analytically, it can be written as (5)

$$
G^{\sigma}\left(x_{V}=x_{0}, T=1750 K\right)=G^{B C C}\left(x_{V}=x_{0}, T=1750 K\right)
$$

Then, the enthalpy jump was taken into account in the phase transition from the BCC phase to the $\sigma$-phase under the assumption that the enthalpy of the transition does not depend on temperature.

$$
\Delta H^{\sigma}\left(x_{0}, 0\right)=\Delta H^{B C C}\left(x_{0}, 0\right)-\Delta H_{\text {congruent }}\left(x=x_{0}=0.517\right) .
$$


From the Sluter's data [8] can be calculated lattice parameters of pure components at oK,. Given the non-variant reaction (Landolt - Borstein New series IV / 19B) for the point of equal concentrations of Fe-V enthalpy of transformation at $T=1514 \mathrm{~K}$ is. Experimental thermodynamic data presented in Table 1, for the BCC phase of Fe-V can be used for the $\sigma$-phase at the equal concentration. Next, we need to go down the temperature of the point of equal concentration to a temperature of $\mathrm{T}=0 \mathrm{~K}$. The enthalpy of transition of $\sigma$-phase in the BCC phase calculated by the formula

$$
\Delta H^{B C C}\left(x_{0}, T=1700\right)=\Delta H^{B C C}\left(x_{0}, 0\right)+\int_{0}^{T=1700} \Delta C_{P}^{B C C}\left(x_{0}, T\right) d T
$$

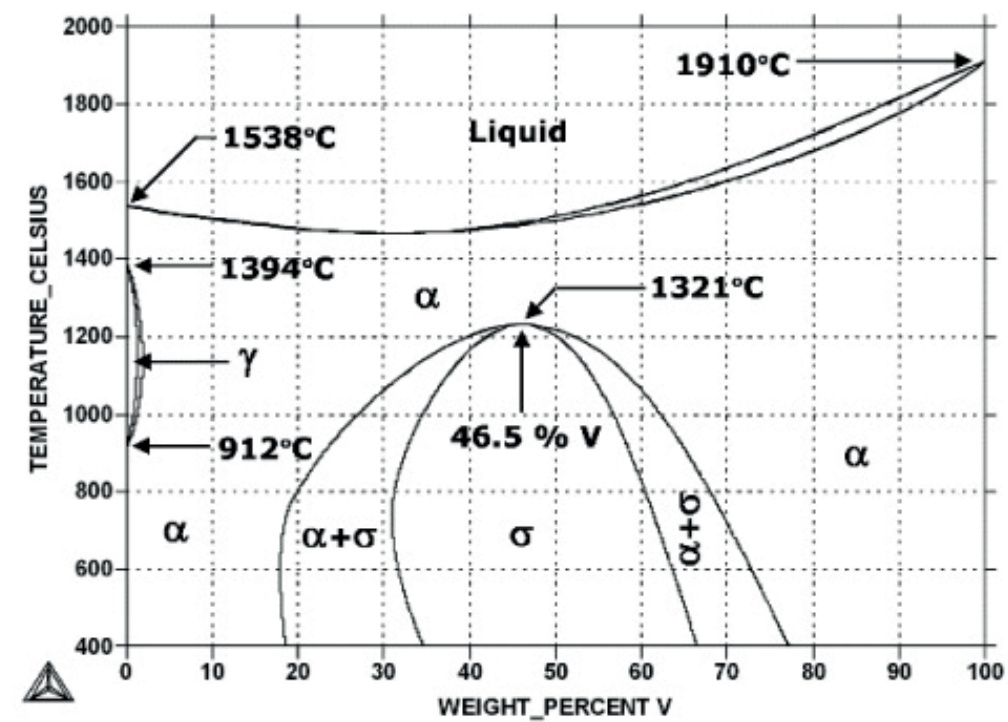

Figure 1: The phase diagram of the Fe-V system [1].

The enthalpy jump at the transition from BCC to $\sigma$-phase, in assumption that the enthalpy of transition is temperature independent, was taken into account as the next step.

$$
\Delta H^{\sigma}\left(x_{0}, 0\right)=\Delta H^{B C C}\left(x_{0}, 0\right)-\Delta H_{\text {congruent }}\left(x=x_{0}=0.517\right)
$$

As a result, the mixing enthalpy dependence on the second element concentrations was plotted by the three points: $\Delta H_{F e}^{\sigma \rightarrow B C C} ; \Delta H_{V}^{\sigma \rightarrow B C C} ; \Delta H^{\sigma}\left(x_{0}, 0\right)$, the difference in the enthalpies of the pure species between the BCC and $\sigma$-phases at $\mathrm{T}=0 \mathrm{~K}$, by least square method approximation to begin searching the system energetic parameters (see Figure 2, table 1).

The magnetic contribution to (1a) was taken into account in the framework of the Inden-Hillert-Jarl formalism [5]. The mean magnetic moment $<\mathrm{m}>$ and Tc dependence on the composition was obtained from an assessment of the experimental data for the mean magnetic moment $<\mathrm{m}>$ and Curie temperature Tc for alloys with the structure of the $\sigma$-phase of the $\mathrm{Fe}-\mathrm{Cr}$ and Fe-V systems. 
Energy parameters of the model were calculated by reference to the energy of formation of ordered complexes $(A, D)_{10}^{12} B_{4}^{15}(C, E)_{16}^{14}$ by using results shown in Figure 3, Table 1. In accordance with these data the set of equations was obtained to find the initial parameters of the model (at o K) (6).

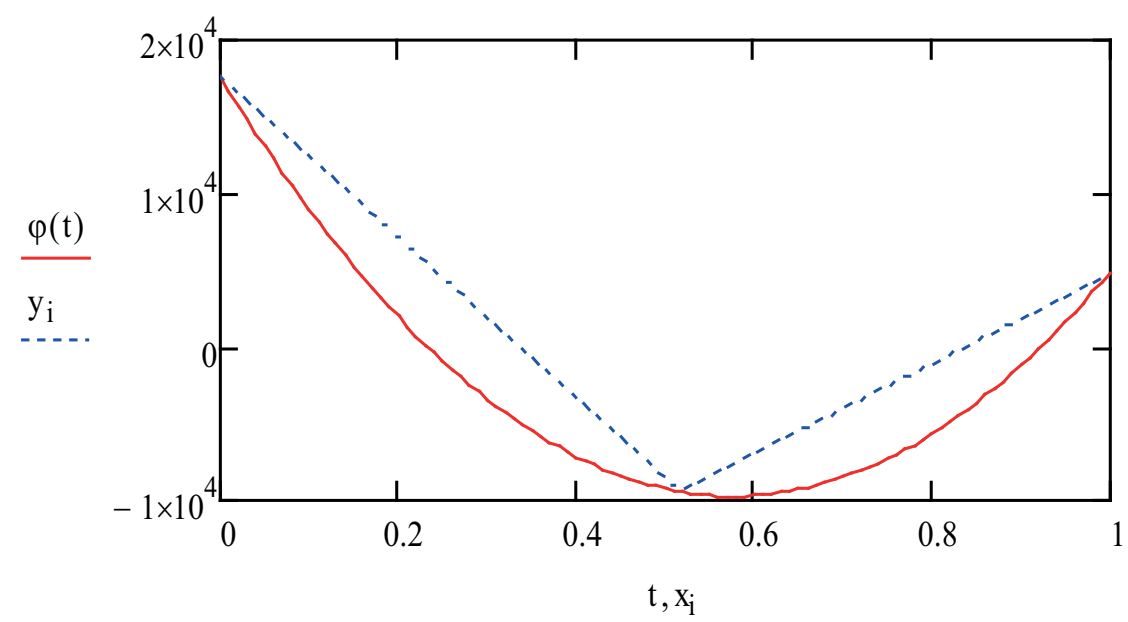

Figure 2: Estimated concentration dependence of enthalpy of mixing $\sigma$-phase relative BCC-phases for pure the two components of the Fe - V system.

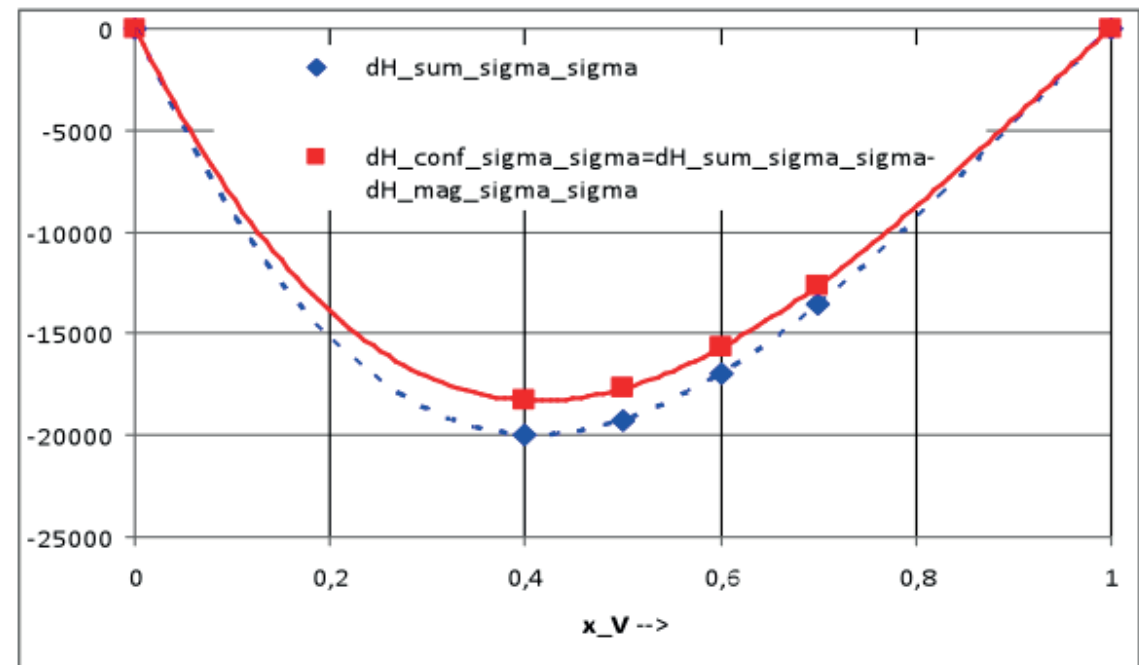

Figure 3: The concentration dependence of the Gibbs energy of mixing relative sigma phases for pure components for the Fe-V system at $\mathrm{T}=\mathrm{oK}$ was obtained of the least square method taking into account the framework of the Inden-Hillert-Jarl formalism for searching initial energetic parameters. 
TABLE 1: The results of calculations of the enthalpy of mixing (Fig.3) for the ordered complexes $(\mathrm{Fe}, \mathrm{V})_{10}^{12}(\mathrm{Fe}, \mathrm{V})_{4}^{15}(\mathrm{Fe}, \mathrm{V})_{16}^{14}$ structure $\sigma$-phase at o $\mathrm{K}$ in the system Fe-V.

\begin{tabular}{|c|c|c|c|c|}
\hline & $(\mathrm{Fe}, \mathrm{V})_{10}^{12}(\mathrm{Fe}, \mathrm{V})_{4}^{15}(\mathrm{Fe}, \mathrm{V})_{16}^{14}$ & & $x(V)$ & $\begin{array}{c}{ }^{\sigma} \Delta^{\sigma} H^{\sigma} \equiv \\
E_{i} \\
\mathrm{~kJ} / \mathrm{mole}\end{array}$ \\
\hline 1 & VFeV & $\begin{array}{l}y_{1}^{12}=0, \quad y_{2}^{12}=1 \\
y_{1}^{15}=1, \quad y_{2}^{15}=0, \quad y_{1}^{14}=0, \quad y_{2}^{14}=1\end{array}$ & 0.867 & -6.337 \\
\hline 14 & $\begin{array}{l}\left(\mathrm{Fe}_{3 / 4} \mathrm{~V}_{1 / 4}\right)\left(\mathrm{Fe}_{1 / 2} \mathrm{~V}_{1 / 2}\right) \\
\left(\mathrm{Fe}_{1 / 2} \mathrm{~V}_{1 / 2}\right)\end{array}$ & $\begin{array}{l}y_{1}^{12}=3 / 4, \quad y_{2}^{12}=1 / 4, \\
y_{1}^{15}=1 / 2, \quad y_{2}^{15}=1 / 2, y_{1}^{14}=1 / 2, \\
y_{2}^{14}=1 / 2\end{array}$ & 0.4166 & -19.96 \\
\hline 16 & $\begin{array}{l}\left(\mathrm{Fe}_{9 / 10} \mathrm{~V}_{1 / 10}\right)\left(\mathrm{Fe}_{7 / 8} \mathrm{~V}_{1 / 8}\right) \\
\left(\mathrm{Fe}_{3 / 4} \mathrm{~V}_{1 / 4}\right)\end{array}$ & $\begin{array}{l}y_{1}^{12}=9 / 10, \quad y_{2}^{12}=1 / 10, \\
y_{1}^{15}=7 / 8, \quad y_{2}^{15}=1 / 8, y_{1}^{14}=3 / 4, \\
y_{2}^{14}=1 / 4\end{array}$ & 0.1833 & -14.47 \\
\hline 18 & $\begin{array}{l}\left(\mathrm{Fe}_{19 / 20} \mathrm{~V}_{1 / 20}\right)\left(\mathrm{Fe}_{15 / 16} \mathrm{~V}_{1 / 16}\right) \\
\left.\mathrm{Fe}_{7 / 8} \mathrm{~V}_{1 / 8}\right)\end{array}$ & $\begin{array}{l}y_{1}^{12}=19 / 20, \quad y_{2}^{12}=1 / 20 \\
y_{1}^{15}=15 / 16, \quad y_{2}^{15}=1 / 16, y_{1}^{14}=7 / 8 \\
y_{2}^{14}=1 / 8\end{array}$ & 0.09167 & -8.55 \\
\hline 21 & $\begin{array}{l}\left(\mathrm{Fe}_{1 / 20} \mathrm{~V}_{19 / 20}\right)\left(\mathrm{Fe}_{1 / 16} \mathrm{~V}_{15 / 16}\right) \\
\left(\mathrm{Fe}_{1 / 8} \mathrm{~V}_{7 / 8}\right)\end{array}$ & $\begin{array}{l}y_{1}^{12}=1 / 20, \quad y_{2}^{12}=19 / 20, \\
y_{1}^{15}=1 / 16, \quad y_{2}^{15}=15 / 16, \quad y_{1}^{14}=1 / 8, \\
y_{2}^{14}=7 / 8\end{array}$ & 0.9083 & -4.399 \\
\hline 22 & $\begin{array}{l}\left(\mathrm{Fe}_{/ 10} \mathrm{~V}_{9 / 10}\right)\left(\mathrm{Fe}_{1 / 8} \mathrm{~V}_{7 / 8}\right) \\
\left(\mathrm{Fe}_{1 / 4} \mathrm{~V}_{3 / 4}\right)\end{array}$ & $\begin{array}{l}y_{1}^{12}=1 / 10, \quad y_{2}^{12}=9 / 10, \\
y_{1}^{15}=1 / 8, \quad y_{2}^{15}=7 / 8, y_{1}^{14}=1 / 4, \\
y_{2}^{14}=3 / 4\end{array}$ & 0.8167 & -8.655 \\
\hline 23 & $\begin{array}{l}\left(\mathrm{Fe}_{1 / 4} \mathrm{~V}_{3 / 4}\right)\left(\mathrm{Fe}_{1 / 2} \mathrm{~V}_{1 / 2}\right) \\
\left(\mathrm{Fe}_{1 / 2} \mathrm{~V}_{1 / 2}\right)\end{array}$ & $\begin{array}{l}y_{1}^{12}=1 / 4, \quad y_{2}^{12}=3 / 4, \\
y_{1}^{15}=1 / 2, \quad y_{2}^{15}=1 / 2, y_{1}^{14}=1 / 2, \\
y_{2}^{14}=1 / 2\end{array}$ & 0.5833 & -17.47 \\
\hline 42 & FeVV & $\begin{array}{l}y_{1}^{12}=1, \quad y_{2}^{12}=0 \\
y_{1}^{15}=0, \quad y_{2}^{15}=1, \quad y_{1}^{14}=0, \quad y_{2}^{14}=1\end{array}$ & 0.667 & -14.84 \\
\hline 46 & $(\mathrm{Fe})(\mathrm{V})\left(\mathrm{Fe}_{3 / 4} \mathrm{~V}_{1 / 4}\right)$ & $\begin{array}{l}y_{1}^{12}=1, \quad y_{2}^{12}=0 \\
y_{1}^{15}=0, \quad y_{2}^{15}=1, \quad y_{1}^{14}=3 / 4 \\
y_{2}^{14}=1 / 4\end{array}$ & 0.267 & -17.88 \\
\hline
\end{tabular}




$$
\begin{aligned}
& \int \frac{109}{120} \delta E_{1}+\frac{19}{20} \delta E_{2}+\frac{7}{8} \delta E_{3}+\left(\frac{109}{120}\right)^{2} \delta E_{4}+\left(\frac{19}{20}\right)^{2} \delta E_{5}+\left(\frac{7}{8}\right)^{2} \delta E_{6} \\
& +\frac{109 \cdot 19}{120 \cdot 20} \delta E_{7}+\frac{109 \cdot 7}{120 \cdot 8} \delta E_{8}+\frac{19 \cdot 7}{20 \cdot 8} \delta E_{9}=E_{21} \\
& \frac{49}{60} \delta E_{1}+\frac{9}{10} \delta E_{2}+\frac{3}{4} \delta E_{3}+\left(\frac{49}{60}\right)^{2} \delta E_{4}+\left(\frac{9}{10}\right)^{2} \delta E_{5}+\left(\frac{3}{4}\right)^{2} \delta E_{6} \\
& +\frac{49 \cdot 9}{600} \delta E_{7}+\frac{49 \cdot 3}{240} \delta E_{8}+\frac{27}{40} \delta E_{9}=E_{22} \\
& \frac{7}{12} \delta E_{1}+\frac{3}{4} \delta E_{2}+\frac{1}{2} \delta E_{3}+\left(\frac{7}{12}\right)^{2} \delta E_{4}+\left(\frac{3}{4}\right)^{2} \delta E_{5}+\left(\frac{1}{2}\right)^{2} \delta E_{6} \\
& +\frac{21}{48} \delta E_{7}+\frac{7}{24} \delta E_{8}+\frac{3}{8} \delta E_{9}=E_{23} \\
& \frac{5}{12} \delta E_{1}+\frac{1}{4} \delta E_{2}+\frac{1}{2} \delta E_{3}+\left(\frac{5}{12}\right)^{2} \delta E_{4}+\left(\frac{1}{4}\right)^{2} \delta E_{5}+\left(\frac{1}{2}\right)^{2} \delta E_{6} \\
& \left\{+\frac{5}{48} \delta E_{7}+\frac{5}{24} \delta E_{8}+\frac{1}{8} \delta E_{9}=E_{14}\right. \\
& \frac{11}{60} \delta E_{1}+\frac{1}{10} \delta E_{2}+\frac{1}{4} \delta E_{3}+\left(\frac{11}{60}\right)^{2} \delta E_{4}+\left(\frac{1}{10}\right)^{2} \delta E_{5}+\left(\frac{1}{4}\right)^{2} \delta E_{6} \\
& +\frac{11}{600} \delta E_{7}+\frac{11}{240} \delta E_{8}+\frac{1}{40} \delta E_{9}=E_{16} \\
& \frac{11}{120} \delta E_{1}+\frac{1}{20} \delta E_{2}+\frac{1}{8} \delta E_{3}+\left(\frac{11}{120}\right)^{2} \delta E_{4}+\left(\frac{1}{20}\right)^{2} \delta E_{5}+\left(\frac{1}{8}\right)^{2} \delta E_{6} \\
& +\frac{11}{2400} \delta E_{7}+\frac{11}{960} \delta E_{8}+\frac{1}{160} \delta E_{9}=E_{18} \\
& \frac{13}{15} \delta E_{1}+\delta E_{2}+\delta E_{3}+\left(\frac{13}{15}\right)^{2} \delta E_{4}+\delta E_{5}+\delta E_{6}+\frac{13}{15} \delta E_{7}+\frac{13}{15} \delta E_{8}+\delta E_{9}=E_{1} \\
& \frac{2}{3} \delta E_{1}+\delta E_{3}+\left(\frac{2}{3}\right)^{2} \delta E_{4}+\delta E_{6}+\frac{2}{3} \delta E_{8}=E_{42} \\
& \frac{4}{15} \delta E_{1}+\frac{1}{4} \delta E_{3}+\left(\frac{4}{15}\right)^{2} \delta E_{4}+\frac{1}{4} \delta E_{6}+\frac{1}{15} \delta E_{8}=E_{46}
\end{aligned}
$$

Solving linear system (7) relatively the energy parameters of 3-sublattice model solutions have been obtained for the nine independent energy parameters. Then, the procedure of the inverse problem solution was conducted (minimization of equation 
(7) in the space of energy parameters) 3SM. Procedure for solving the inverse problem (minimization of equation (8) in the space of energy parameters) 3PM was carried out to correction the energy settings found under the condition of the local and global stabibility (3). The solving the problem of correcting the initial parameters of the model using by the normalization condition (8). Illustration of stable and metastable solutions are Figure 4,5.

$$
\chi^{2}=\sum_{i=1}^{N}\left[\frac{{ }^{\sigma} \Delta^{\sigma} H_{\exp }^{\sigma}\left(x_{i}\right)-{ }^{\sigma} \Delta^{\sigma} H_{\text {calc }}^{\sigma}\left(x_{i}\right)}{\delta \Delta H^{\sigma}\left(x_{i}\right)}\right] /(N-8) \rightarrow 0, \quad N=11, \frac{\delta \Delta H_{\exp }^{\sigma}\left(x_{i}\right)}{\Delta H_{\exp }^{\sigma}\left(x_{i}\right)} \approx 0.1
$$

The optimizing energy values of the model parameters for $\sigma$ - phase are shown in table 2

Illustration of stable and metastable solutions are Figure 3,4.

$$
{ }^{f \sigma} \Delta^{p \sigma} G^{\sigma}\left(x_{V}=0, y_{2}^{12}, y_{2}^{15}, T\right)={ }^{f \sigma} \Delta^{p \sigma} G^{\sigma}\left(x_{V}=1, y_{2}^{12}, y_{2}^{15}, T\right)=0
$$

TABLE 2: The optimize energy values of the model parameters for $\sigma$-phase $(\mathrm{kJ} / \mathrm{mol})$.

\begin{tabular}{c|c|c|}
$\Delta E_{1}=-5.598 \cdot 10^{2}$ & $\Delta E_{2}=2.007 \cdot 10^{2}$ & $\Delta E_{3}=2.798 \cdot 10^{2}$ \\
$\Delta E_{4}=4.073 \cdot 10^{3}$ & $\Delta E_{5}=0.715 \cdot 10^{3}$ & $\Delta E_{6}=0.910 \cdot 10^{3}$ \\
$\Delta E_{7}=-3.285 \cdot 10^{3}$ & $\Delta E_{8}=-3.737 \cdot 10^{3}$ & $\Delta E_{9}=1.450 \cdot 10^{3}$
\end{tabular}

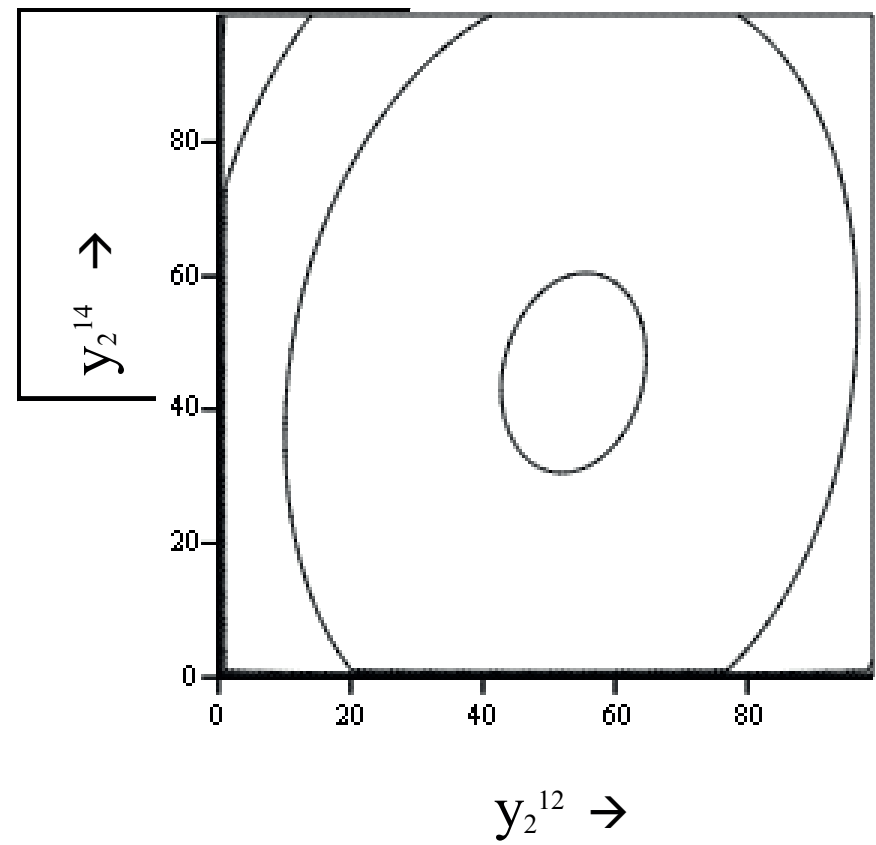

Figure 4: Illustration of stable solutions $y_{2}^{12}, y_{2}^{14}$, multiplied by 100 . 


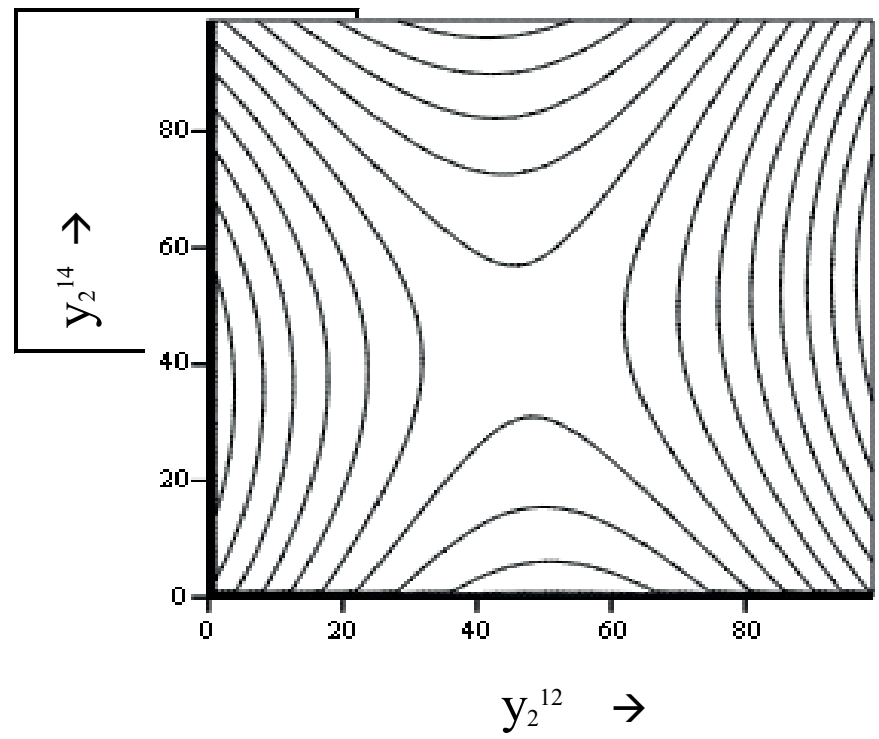

Figure 5: Illustration of metastable solutions, multiplied by 100.

\section{The calculation results}

According to $3 \mathrm{SM}$, calculations of the concentration dependences of the vanadium atom distribution over the three model sublattices with coordination of neighbors atoms 12,15 and 14 calculated for the Fe-V system at $T=$ oK were made (see Figure 6). A comparison of the calculations of the $\sigma \mathrm{Fe}-\mathrm{Cr}$ alloys [1] with the $\sigma \mathrm{Fe}-\mathrm{V}$ alloys obtained in 3PM, was made (Figures 6-7). Comparing Figures 6 and 7 one can notice that in both cases in iron-rich alloys, the impurity atoms $(\mathrm{V}, \mathrm{Cr})$ of the sub-lattice are filled first with atoms whose coordination environment equals to 15 . The concentration dependence of the Gibbs potential of the Fe-V system at $T=$ oK was calculated, see in Figure 7.

The consistency of the input data and the calculation results for the thermodynamic properties (see Figures 2 and 8 ) is obtained, namely, the dependence of the enthalpy of mixing of the $\sigma$-phase of the Fe-V system on the composition relatively of the BCC phases of pure Fe and V (Figs. 2 and 8). The beats in Fig.8 are due to the influence of errors calculated both by the values of the energy parameters of the model and by the values of the mole fractions of the concentrations of vanadium atoms populating three model sub-lattices of the $\sigma$-phase.

The input data was used to find the initial values of the model parameters. The consistency of these data proves the correctness of the found energy parameters of the model. 


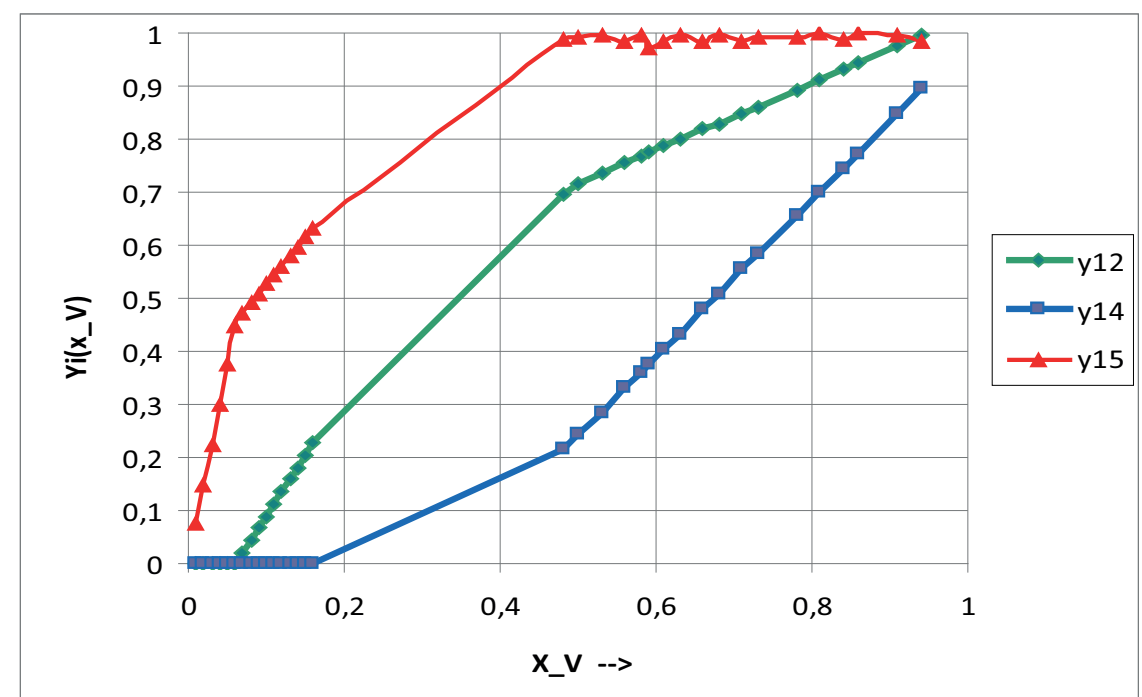

Figure 6: The concentration dependence of the distribution of vanadium atoms in three model sub-lattices with the coordination of atoms - the neighbors of 12,14 and 15 , calculated at oK.

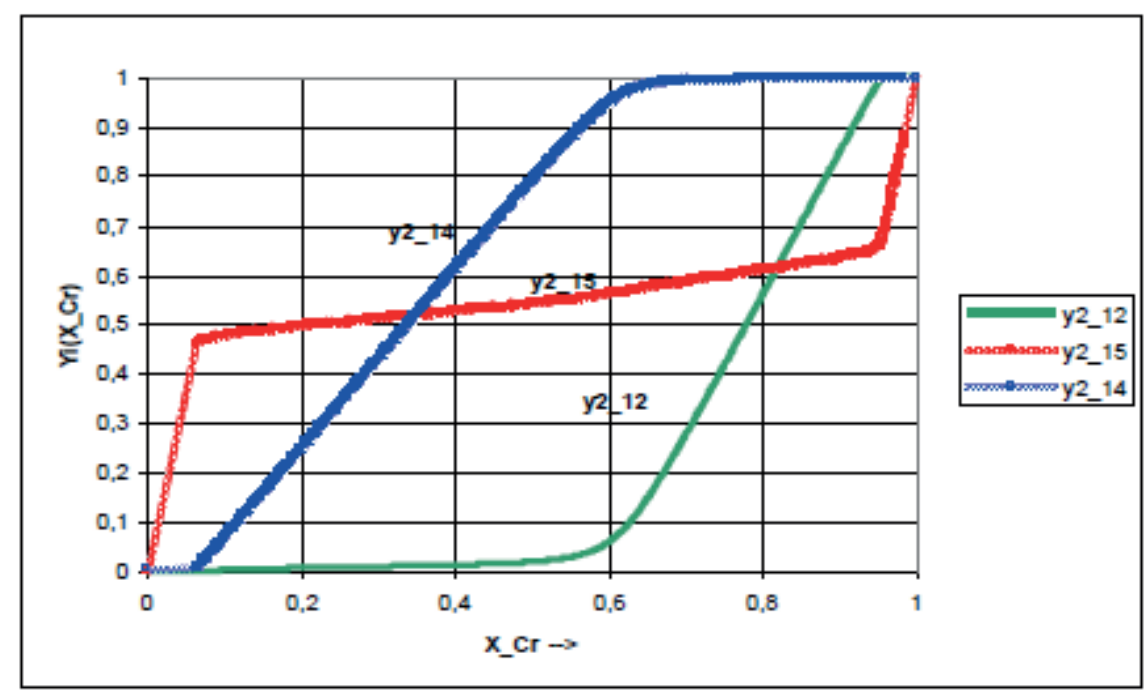

Figure 7: The concentration dependence of the distribution of cromium atoms in three model sub-lattices with the coordination of atoms - the neighbors of 12,14 and 15 , calculated at oK $[1,2]$.

\section{Conclusion}

In this work it was possible to calculate the energy mixing for $\sigma$-phase as function of composition at $\mathrm{T}=\mathrm{oK}$, despite the absence of any ab-initio calculated data of a mixing enthalpy of the $\sigma \mathrm{Fe}-\mathrm{V}$ alloys, in contrast to $[1,2]$. The distributions of atomic species in different model sub-lattices and the TPs depending on the $\sigma$ Fe-V alloy compositions at $\mathrm{T}=\mathrm{oK}$ were evaluated with the help of energy and magnetic parameters (Curie temperature and mean magnetic moment) which were previously calculated, and by solving the set of equations at $\mathrm{T}=\mathrm{oK}$ obtained by minimizing the functional $\Delta \mathrm{G}$ with 


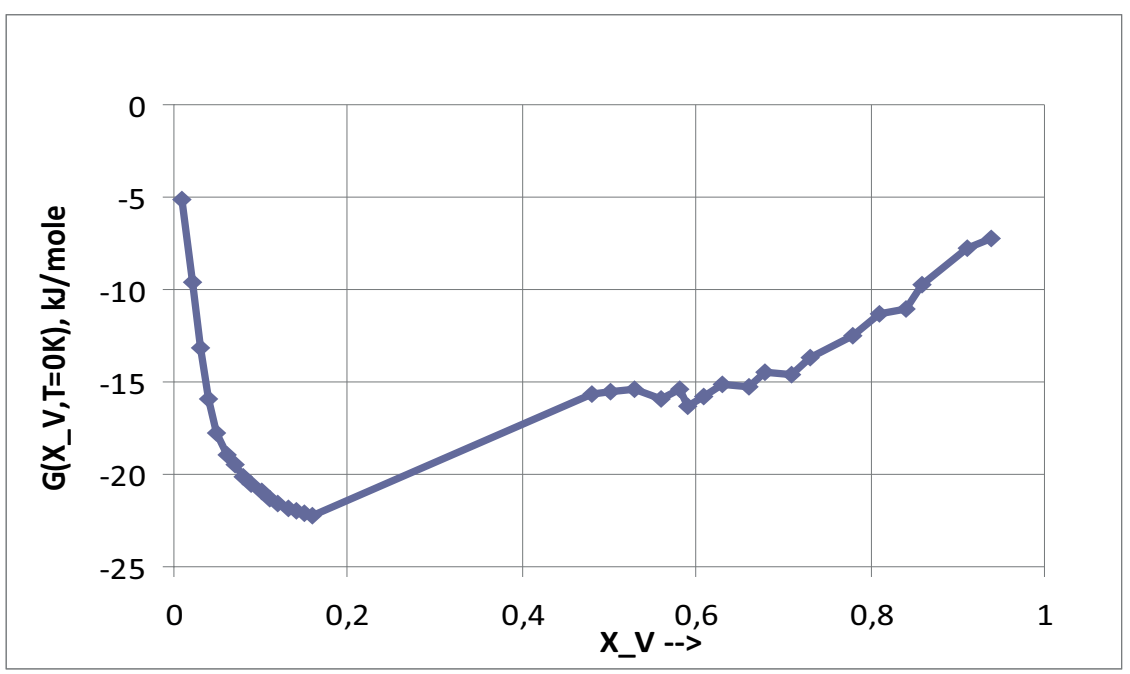

Figure 8: The concentration dependence of the configurational part of Gibbs energy of mixing for $\sigma$-phase of the Fe-V system at $\mathrm{T}=\mathrm{oK}$.

respect to the independent configuration degrees of freedom. The comparison of the calculated and experimental data on the structural properties of $\sigma$-phases in $\mathrm{Fe}$ - $(\mathrm{Cr}$, v) systems depending on composition and temperature was conducted.

\section{Acknowledgements}

Research supported by grants of RFBR 13-03-00462-a and Department of Chemistry and Materials Science of Presidium RAS (grant OKhNM-02).

\section{References}

[1] A.Udovsky, M.Kupavtsev. J.Min.Metall. Sect.B-Metall., 48 (3) (2012) $483-489$.

[2] A.Udovsky, M.Kupavtsev. IOP Conf.Series: Materials Science and Eng., 130 (1) (2016) 012064-1-012064-6

[3] J. Cieslak, B.F.O.Costa, St.M.Dubiel et all. Journal of MMM, 321(2009) 2160

[4] M. Sluter, CALPHAD,.30 (2006) 357-366.

[5] A.T.Dinsdale. CALPHAD, 17 (4) (1991) 317 - 425.

[6] E. Kabliman, A.V. Ruban, P. Blaha at all. Appl. Sci., 2 (2012) 654-668

[7] J.Havrankova, J.Vrestal, L.J.Wang, M.Sob, Phys. Rev. B, 63 (2001) 174104 